\title{
Working Memory Training Is Associated with Changes in Resting State Functional Connectivity in Children Who Were Born Extremely Preterm: a Randomized Controlled Trial
}

\author{
Chieh-En Jane Tseng ${ }^{1}(1) \cdot$ Leona Pascoe $^{2} \cdot$ Gehan Roberts $^{3,4,5} \cdot$ Lex W. Doyle $^{3,4,6} \cdot$ Katherine J. Lee $^{3,4}$. \\ Deanne K. Thompson ${ }^{3,4,7} \cdot$ Marc Seal $^{3,4} \cdot$ Elisha K. Josev $^{3,4}$ • Jian Chen ${ }^{3,8} \cdot$ Chiara Nosarti $^{1,9} \cdot$ Peter J. Anderson ${ }^{10}$
}

Received: 27 March 2019 / Accepted: 20 August 2019 / Published online: 13 September 2019

(C) The Author(s) 2019

\begin{abstract}
Children born extremely preterm (EP; $<28$ weeks of gestation) or extremely low birth weight (ELBW; $<1000 \mathrm{~g})$ are at increased risk of working memory deficits compared with their term-born peers and may benefit from working memory training. This study aimed to determine whether Cogmed Working Memory Training®, compared with a placebo training program, was associated with changes in resting-state functional connectivity (rsfc) and whether these changes correlated with working memory performance in EP/ELBW children. Twenty-one 7-year-old EP/ELBW children were enrolled in a double-blinded randomized controlled trial and had magnetic resonance imaging (MRI) assessments (Cogmed, $n=12$; placebo (a non-adaptive version of Cogmed), $n=9$ ). Prior to training (baseline) and 2 weeks post-training, all children received a cognitive assessment, inclusive of immediate memory and working memory measures and an MRI. The Cogmed Improvement Index was used as a measure of improvement in trained activities in the Cogmed group. Resting-state functional MRI was used to measure training-related changes in intra- and internetwork rsfc. The networks assessed include the default mode network, the left and right central executive networks, the bilateral executive network, the dorsal attention network, and the salience network. rsfe data were compared between treatment groups and investigated in relation to changes in working memory performance. There was little evidence of differences in intra- or internetwork rsfe strength changes from baseline to post-training between treatment groups. In the Cogmed group, working memory performance was associated with increased rsfc from baseline to post-training within the precuneus network, but not in the placebo group. In the Cogmed group, results that did not survive multiple comparison correction further showed that improvement in trained activities was associated with increased rsfc between the left central and bilateral executive networks, and with decreased rsfc within the right central executive network and between the right central executive and salience networks. Changes in rsfe may facilitate working memory performance following Cogmed training. Further studies are needed to investigate how changes in rsfe are associated with behavioral changes to better support working memory in vulnerable groups.
\end{abstract}

Chiara Nosarti and Peter J. Anderson are joint senior authors.

Electronic supplementary material The online version of this article (https://doi.org/10.1007/s41465-019-00150-7) contains supplementary material, which is available to authorized users.

Chieh-En Jane Tseng

ctseng4@mgh.harvard.edu

1 Department of Psychosis Studies, Institute of Psychiatry, Psychology and Neuroscience, King's College London, London, UK

2 Turner Institute for Brain and Mental Health, Monash University, Melbourne, Australia

3 Murdoch Children's Research Institute, Melbourne, Australia

4 Department of Paediatrics, The University of Melbourne, Melbourne, Australia

5 Royal Children's Hospital, Melbourne, Australia

6 Department of Obstetrics and Gynaecology, Royal Women's Hospital, The University of Melbourne, Melbourne, Australia

7 Florey Institute of Neuroscience and Mental Health, Melbourne, Australia

8 Department of Medicine, Monash University, Melbourne, Australia

9 Centre for the Developing Brain, Department of Perinatal Imaging and Health, Division of Imaging Sciences and Biomedical Engineering, King's College London, London, UK

10 Monash Institute of Cognitive and Clinical Neurosciences, Monash University, Melbourne, Australia 
Keywords Extremely preterm $\cdot$ Working memory training $\cdot$ Resting-state fMRI

\section{Introduction}

In recent years, much attention has focused on training the developing brain (see Jolles and Crone 2012 for a review), with the rationale being that training specific cognitive functions (i.e., attention and working memory) during development, can offer advantages in terms of learning capacity. Cognitive training interventions in children with neurodevelopmental disorders have demonstrated gains on trained and/or non-trained tasks (Kerns et al. 2017; Robinson et al. 2014). It is possible that neuroplasticity, which refers to the life-long process by which the brain adapts to changes in its environment (Pascual-Leone et al. 2005), may underlie these cognitive improvements. Training in adults primarily reshapes the existing neural landscape, while training in young children may influence the establishment of developing neural networks. It has therefore been suggested that childhood may be a sensitive period during which training has its largest effects (Jolles and Crone 2012).

Working memory has been the focus of cognitive training interventions in children and adolescents born very preterm $(<32$ weeks of gestation) (Grunewaldt et al. 2013; Lohaugen et al. 2011; Everts et al. 2015), given they often report working memory problems (see Anderson 2014 for a review), which are particularly pronounced in those individuals born extremely preterm (EP; $<28$ weeks of gestation) (Marlow 2004). Working memory problems are thought to underlie poor academic achievement in young people (Simone et al. 2017), which in turn affect their social and economic prospects later in life (Levine and Nourse 1998; Heath and Ross 2000). Previous working memory training studies in young preterm individuals have been conducted at preschool age (Grunewaldt et al. 2013), when cognitive resources to support training are not well developed, and in adolescence (Lohaugen et al. 2011), when working memory capacities are approaching adult levels, with potentially little scope for improvement (Jolles and Crone 2012; Gathercole 1999). In contrast, early school age may be a preferable period to train working memory, given that by this age, children have developed sufficient resources to support maturing working memory functioning. Further, at this age, brain regions that are involved in working memory (i.e., frontal and parietal cortices) are sufficiently developed to perform working memory tasks but are still maturing and can be influenced by the environment (through experience, exposure, and training), thereby allowing targeted interventions to utilize their residual neuroplastic capacity (Johnson 2011).

Resting-state functional magnetic resonance imaging (rs-fMRI) is a fast, task-independent method for exploring the brain's functional organization. The functional networks identified at rest closely correspond to networks that are active during the performance of specific tasks (Smith et al. 2009) and can predict task-induced brain activity or an individual's task performance (Mennes et al. 2010; Zou et al. 2013). Most importantly, resting-state functional connectivity (rsfc) has been found to change as a result of learning (Lewis et al. 2009). In relation to working memory, results of previous studies showed that working memory performance was related to rsfc within central executive and salience networks (Engstrom et al. 2013), and within the precuneus/posterior cingulate cortex of the default mode network (Sala-Llonch et al. 2012). However, to date, no papers have examined rsfe in relation to working memory training in EP children and/or extremely low birth weight (ELBW; < $1000 \mathrm{~g}$ birth weight). Studying traininginduced rsfc changes in relation to training outcomes may help to increase understanding of the neural mechanisms associated with working memory in EP/ELBW children and aid in the refinement of interventions aimed at enhancing working memory capacity.

The current study used rs-fMRI to investigate traininginduced neuroplasticity following a working memory training program (Cogmed Working Memory Training $®$ ) compared with a placebo training program in 7-year-old EP/ ELBW children. Participants were part of a larger, doubleblind randomized controlled trial. In the main trial, we failed to identify benefits of Cogmed on working memory up to 24 months post-training compared with the placebo program (Anderson et al. 2018); however, fMRI is powerful in detecting changes in hemodynamic responses even in the absence of observable effects on cognitive performance (Froudist-Walsh et al. 2015; Erk et al. 2011). We therefore hypothesized that training-induced changes in rsfc would be greater in the Cogmed group compared with the placebo group. We further explored whether traininginduced changes in rsfe were associated with traininginduced changes in cognitive performance, and whether this differed between treatment groups. We hypothesized that training-induced rsfc would be associated with cognitive improvements, regardless of group. Finally, associations between training-induced rsfc changes and improvements during the working memory training were explored within the Cogmed group. We hypothesized that greater traininginduced rsfc changes would be associated with greater working memory training gains. These hypotheses are based on specific adaptive training effects previously reported that suggest a central role of load adaptation to support effective working memory gains (Klingberg et al. 2002, 2005; Holmes et al. 2009). 


\section{Materials and Methods}

\section{Study Participants}

Participants in the current study were born at either $<28$ completed weeks of gestation and/or with birth weights $<1000 \mathrm{~g}$ in the state of Victoria, Australia, in 2005 (Doyle et al. 2011, 2010), and were enrolled in a double-blinded, placebocontrolled randomized clinical trial of Cogmed Working Memory Training® (hereafter referred to as Cogmed) following parental consent $(n=91)$. The children were randomized to either the Cogmed training program group or the placebo group in a 1:1 ratio. The randomization schedule was generated by a biostatistician (K. L.) who was independent of the study, using block randomization with variable block sizes. The project coordinator (L. P.) and a research assistant managed the randomization. The participant and their family remained blinded to the assigned program allocation throughout the study. Other than the project coordinator and research assistant, all other members of the trial team were blinded to program allocation throughout the study.

Children were excluded if they had a severe intellectual, sensory, or physical impairment that affected their capacity to attend mainstream school ( $n=16)$, were from families considered unable to comply with the training schedule $(n=2)$, or were not suitable for the study due to location or previous training exposure $(n=3)$. Of the 91 children enrolled in the trial, 42 children consented to optional MRI, completed working memory assessments, and had successful MRI scans both before (baseline) and 2 weeks after training (post-training). Loss of data included non-consent to the MRI, noncompliance to the MRI procedures, incomplete scans, and failure to return for follow-up neuroimaging. Of the 42 children who completed the working memory training baseline and post-training scans, 19 children were excluded due to excessive motion $(>0.35 \mathrm{~mm}$ relative mean displacement, approximately $0.7 \mathrm{~mm}$ frame displacement) for at least one time point, one child was excluded due to having a poor-quality $T_{1^{-}}$weighted image, and one child due to having dental braces which caused artifacts on the images. Therefore, the rsfc analyses were performed in 21 children with MRI data at both time points $($ Cogmed $=12$; placebo $=9)$.

\section{Cogmed Working Memory Training}

Children were randomly assigned to receive either Cogmed or a placebo program. Cogmed includes a range of different computerized working memory activities that are practiced intensively ( 5 days per week for 5 to 7 weeks). The program is adaptive, such that the complexity level of individual activities varies with the child's current working memory capacity on a trial-by-trial basis. The placebo program is identical to Cogmed, except that it is non-adaptive, with the complexity of activities set to an undemanding level throughout the training period. This design enabled us to explore possible neuroplastic changes specifically associated with adaptive working memory training (Anderson and Doyle 2008). Children attended assessments at the Royal Children's Hospital (RCH), Melbourne, Australia, at both baseline and 2 weeks post-training.

\section{MRI Acquisition}

MRI at both baseline and post-training was undertaken without sedation using a Siemens 3 Tesla Magnetom Trio scanner and a 32-channel head coil at the RCH. Children underwent a 3D $T_{1}$-weighted magnetization-prepared rapid gradient-echo (MP-RAGE) sequence $(0.85 \mathrm{~mm}$ sagittal slices, in-plane resolution $0.82 \mathrm{~mm}^{2}$, flip angle $9^{\circ}$, repetition time $1900 \mathrm{~ms}$, echo time $2.27 \mathrm{~ms}$, field of view $210 \times 210 \mathrm{~mm}$, matrix $256 \times 256$ ) and a rs-fMRI echo planar imaging sequence with prospective acquisition correction (PACE) $(4.0 \mathrm{~mm}$ axial slices, in-plane resolution $=3.3 \mathrm{~mm}^{2}$, flip angle $=90^{\circ}$, repetition time $=$ $2400 \mathrm{~ms}$, echo time $=35 \mathrm{~ms}$, field of view $=210 \times 210 \mathrm{~mm}$, matrix $=64 \times 64$, scan duration $=10 \mathrm{~min}$ ).

\section{Imaging Analysis}

The researchers who analyzed the imaging data (C.E. J. T. and $\mathrm{C}$. N.) were blinded regarding group assignment when conducting the imaging analysis. A customized template was created from the structural images of the 21 included children using Advanced Normalization Tools (ANTs; http://stnava.github.io/ANTs/). The resting-state fMRI preprocessing steps were performed using Functional Magnetic Resonance Imaging of the Brain (FMRIB) Software Library (FSL; www.fmrib.ox.ac.uk/fsl) and included non-brain removal using Brain Extraction Tool, motion correction using FMRIB's Linear Image Registration Tool (Jenkinson et al. 2002), temporal filtering, slice-timing correction, spatial smoothing, normalization to the custom template using FMRIB's Linear Image Registration Tool (Jenkinson et al. 2002; Jenkinson and Smith 2001) and FMRIB's Nonlinear Image Registration Tool (Andersson et al. 2010), and denoising using FMRIB's independent component analysis-based Xnoiseifier (Salimi-Khorshidi et al. 2014; Griffanti et al. 2014). Group independent component analysis (ICA) was performed on the whole dataset $(n=21)$ using Group ICA of fMRI Toolbox (GIFT; http://icatb.sourceforge.net). GIFT uses a temporal concatenation approach, during which data reduction is performed via multi-staged principal component analysis and aggregation to generate common-group maps. These components are then back-projected onto each individual's data to create subject-specific spatial maps with corresponding time courses (Calhoun et al. 2008). ICA was 
performed using the Infomax algorithm (Bell and Sejnowski 1995), and repeated 5 times with Icasso (Himberg et al. 2004) to maximize the stability of the derived components. Functional region of interests from the Functional Imaging in Neuropsychiatric Disorders lab (Shirer et al. 2012) and meta-analysis maps using the term "frontoparietal" from http://neurosynth.org were used as templates to identify components as resting-state networks. The FSL tool fslcc was used to find the component that had the highest cross-correlation with each functional network template. This component was visually inspected and determined as the network component if appropriate; if not, the component with the second highest cross-correlation was visually inspected. All networks were identified within the first two components. The following resting-state networks were identified: the salience network ( $\mathrm{SN}$; bilateral anterior cingulate cortex, middle frontal gyrus, insula, putamen, and thalamus), default mode network (DMN; bilateral medial prefrontal cortex, angular gyrus, posterior cingulate cortex, and hippocampus), dorsal attention network (DAN; bilateral inferior frontal gyrus, precentral gyrus, intraparietal sulcus, and lateral occipital cortex), precuneus network (PN; bilateral angular gyrus, posterior cingulate cortex, and precuneus), bilateral executive network (BEN; bilateral middle frontal gyrus, anterior cingulate cortex, posterior cingulate cortex, superior parietal lobule, supramarginal gyrus, and left superior frontal gyrus and inferior temporal gyrus), and left and right central executive network (LCEN; left superior, middle, and inferior frontal gyrus, precuneus, and bilateral anterior and posterior cingulate cortex, RCEN; right middle and inferior frontal gyrus, angular gyrus, and supramarginal gyrus) (Fig. 1). Each identified network was represented by one ICA component; therefore, a total of seven components were included in the analysis. These resting-state networks were chosen because they have previously been identified as relating to working memory performance (Engstrom et al. 2013; Sala-Llonch et al. 2012; Hampson et al. 2006; Charroud et al. 2016; Fang et al. 2016). The strength of connectivity within individual networks was derived from the $z$-scored spatial maps and will be referred to as intra-network $r s f c$. The strength of connectivity between two networks (as estimated by the Pearson's correlation coefficient between pairs of timecourses) will be referred to as inter-network $r s f c$. Subject-specific timecourses were detrended, despiked, and filtered using a fifth-order Butterworth low-pass filter with a high frequency cutoff of $0.15 \mathrm{~Hz}$. All correlation coefficients were transformed to z-scores using Fisher's transformation.

The resting-state networks identified in this study were generated in the customized template space but are represented here on an MNI template with corresponding MNI coordinates.

\section{Cognitive Measures}

At both assessments, children completed selected subtests from the Working Memory Test Battery for Children (WMTB-C) (Pickering and Gathercole 2001), namely Digit Recall, Word List Recall, Block Recall, Mazes Memory, and Backward Digit Recall. The Mister X subtest was completed from the Automated Working Memory Assessment (AWMA) (Alloway 2007). These measures were combined into two composite scores to represent immediate memory and working memory. The immediate memory composite score was a sum of the standardized scores of all WMTB-C subtests except the Backward Digit Recall subtest. The standardized scores of the Backward Digit Recall and Mister X subtests were summed to obtain the working memory composite score. The Cogmed Improvement Index score was used as a measure of improvement on the trained activities, which was available for the Cogmed group only. This score was obtained by calculating the difference between the Start index (i.e., the mean of the three best successful trials on days 2 and 3) and the Max index (i.e., the mean of the three best successful trials on the two best training days). All assessments were conducted blinded to knowledge of treatment group allocation by trained research assistants and psychologists at the Murdoch Children's Research Institute in Melbourne, Australia.

\section{Statistical Analysis}

Training-induced changes (from baseline to post-training) in rsfe and memory composite scores were compared between the Cogmed and placebo groups, adjusting for corresponding baseline values of the measure of interest (e.g., participants' change in Digit Recall was adjusted for their Digit Recall baseline scores). To adjust for baseline values, robust regression and a logistic weight function were used; residuals were then used for independent $t$ tests and subsequent analysis. The association between training-induced changes in intra-/inter-network rsfc (predictor) and changes in memory composite scores (outcome) were explored using linear regression. We investigated whether these associations differed between treatment groups by testing for an interaction between treatment group and rsfc change in the linear regression models. Within the Cogmed group, additional analyses were performed to explore the association between training improvements and changes in rsfc. Results were corrected for multiple comparisons using the BenjaminiHochberg approach (Benjamini and Hochberg 1995). Findings that do not survive multiple comparison correction are referred to as "findings at a trend-level". 


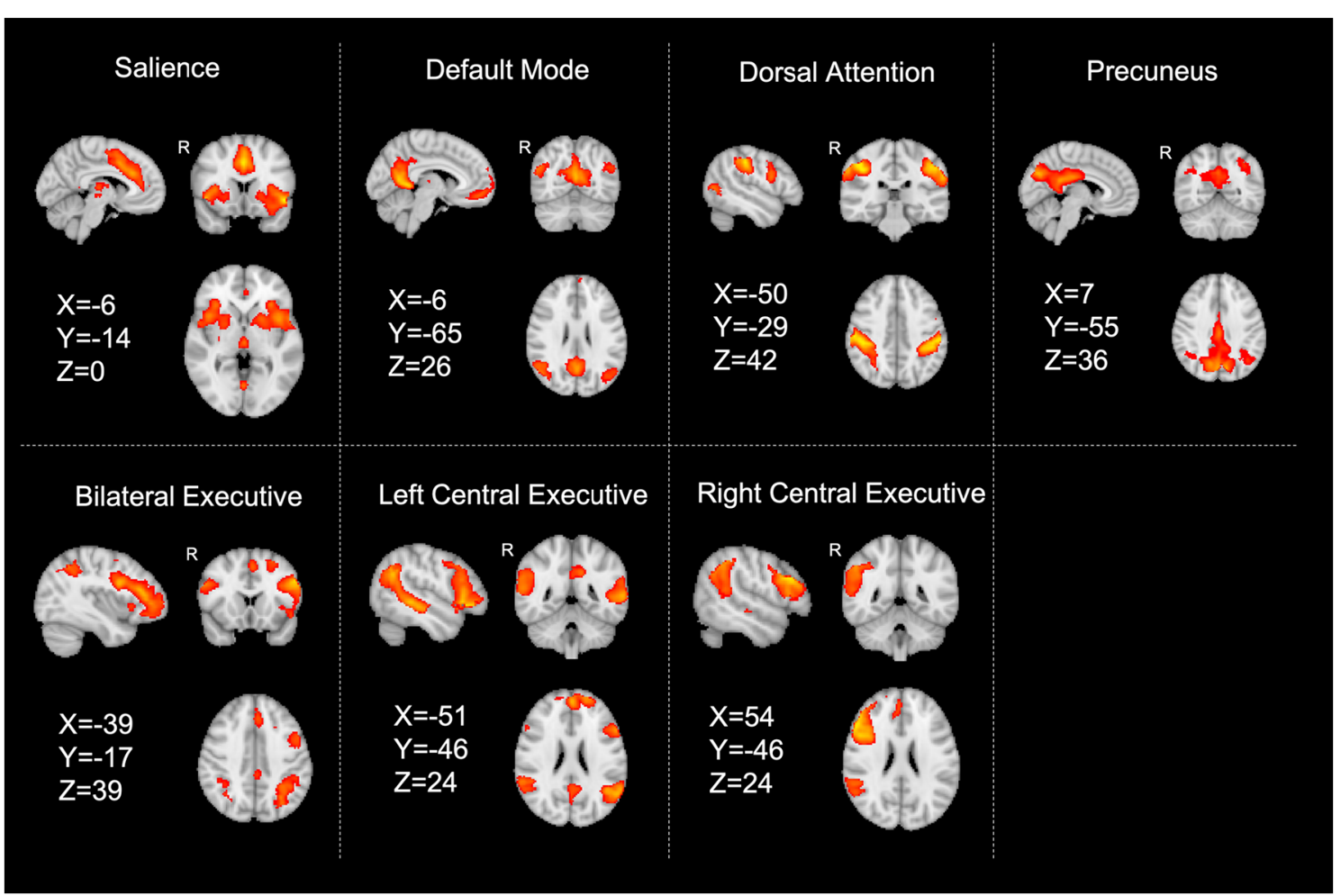

Fig. 1 Resting-state networks of interest

\section{Results}

Study participants $(n=21)$ and non-participants $(n=70)$ had similar characteristics in terms of sex, gestational age, and birth weight; however, participants were slightly older in age compared with non-participants (participants: mean age \pm standard deviation $=7.7 \pm 0.6$; non-participants: mean age \pm standard deviation $=7.2 \pm 0.4$ ). Demographic and neonatal characteristics of the study participants are shown in Table 1. As expected, the two groups were similar with regard to their baseline characteristics.

There was little evidence (i.e., we were unable to reject the null hypothesis of prior to multiple comparison correction) of a difference in the changes in intra-network rsfc (Table 2), inter-network rsfc (Table 3), and immediate memory or working memory (Table 4), from baseline to post-training between the two treatment groups (aim 1). Changes in intra-network rsfc in DAN and DMN were greater in the Cogmed group compared with the placebo group at a trend-level (Table 2 and Fig. 2). Positive scores indicate higher functional connectivity at follow-up than at baseline, while negative scores indicate higher functional connectivity at baseline than at follow-up.

There was evidence that precuneus intra-network rsfc changes were associated with working memory composite score changes in the Cogmed group (regression coefficient $(95 \% \mathrm{CI})=5.92(2.31,9.53), p=0.004)$, but not in the placebo group (regression coefficient $(95 \% \mathrm{CI})=-0.69(-3.69$,
2.32), $p=0.60$ ) (interaction $p=0.05$, Fig. 3). There was little evidence of associations between intra- or inter-network rsfc changes and working memory changes for the other networks investigated. There was also little evidence of any association between changes in intra- or inter-network rsfc and immediate memory composite scores.

In terms of training improvement in the Cogmed group, findings showed associations between higher Cogmed Improvement Index scores, which reflect the capacity to improve across training trials, and decreased baseline to posttraining rsfc change within the RCEN (intra-network) rsfc (regression coefficient $(95 \% \mathrm{CI})=-17.17(-29.38,-4.96)$, $p=0.011$ ), and between the RCEN and the SN (inter-network) rsfc (regression coefficient $(95 \% \mathrm{CI})=-17.15(-29.62,-$ $4.69), p=0.012$ ) at a trend-level, as well as increased baseline to post-training rsfc change between the LCEN and the BEN $($ regression coefficient $(95 \% \mathrm{CI})=19.29(-0.32,38.90), p=$ 0.053 ) at a trend-level (Fig. 4). There was little evidence of associations between intra- or inter-network rsfe change and Cogmed training improvement for any of the other networks investigated.

\section{Discussion}

Our findings demonstrated that changes in working memory and rsfc in EP/ELBW children from baseline to post-training were not different between the Cogmed and placebo groups. 
Table 1 Demographic and neonatal characteristics of the study participants

\begin{tabular}{llllll}
\hline & Cogmed $(n=12)$ & Placebo $(n=9)$ & Cohen's $d$ & $t$ & $p$ value \\
\hline Age at assessment & $7.7 \pm 0.5$ & $7.8 \pm 0.8$ & 0.16 & 0.38 & 0.71 \\
Sex $(\mathrm{M} / \mathrm{F})$ & $4 / 8$ & $3 / 6$ & & & \\
Gestational age $(\mathrm{M} \pm \mathrm{SD})$ & $27.4 \pm 2.7$ & $26.4 \pm 1.1$ & 0.51 & -1.09 & 0.07 \\
Birth weight $(\mathrm{M} \pm \mathrm{SD})$ & $866 \pm 128$ & $974 \pm 274$ & 0.50 & 1.21 & 0.06 \\
\hline
\end{tabular}

$M$ mean, $S D$ standard deviation
However, differences between groups were found in terms of the relationship between training-induced working memory and rsfc changes. Evidence of an association between changes in working memory and training-induced rsfe changes within the precuneus network was observed in the Cogmed group, but not in the placebo group. In relation to improvements on trained activities for the Cogmed group, associations were found for decreased rsfc within the right central executive network and between the right central executive and precuneus networks and increased rsfc between the left central executive and bilateral executive networks at a trend-level.

In the Cogmed group, we found an association between working memory change and rsfc connectivity change in the precuneus network. Greater improvement in working memory performance was mostly associated with increased positive rsfc change. The precuneus has previously been suggested to play a central role in performing tasks that involve first-person perspective, such as visuospatial imagery, self-referential processing, and episodic memory retrieval (Cavanna and Trimble 2006). It is also thought to have the highest resting metabolic rate within the DMN and is extensively connected to cortical and subcortical regions, suggesting a role in integrating both internally and externally driven information (Cavanna and Trimble 2006). For instance, increased rsfc has been previously reported between the precuneus and the DMN at rest, and between the precuneus and the right frontoparietal network during completion of a decision-making task (Utevsky et al.
2014). The observed association between greater precuneus intra-network rsfc change and greater working memory improvements following training in this study may be indicative of improved information integration through the precuneus. Similarly, a previous study showed that training-induced hemodynamic response changes in the precuneus during a cognitive training video game predicted performance improvement on a non-trained working memory task post-training in young healthy adults (aged 22, $n=45$ ) (Nikolaidis et al. 2014). The authors suggested that improvement on tasks similar to training tasks or activities (referred to as near transfer) occurs when training-induced plasticity is evident in brain regions that are implicated in the non-trained task (Nikolaidis et al. 2014). Another study in young adults (aged $22, n=15$ ) reported that working memory improvements after practicing a working memory task (for 6 weeks) were associated with increased frontoparietal connectivity (including the precuneus in the parietal region), and decreased DMN connectivity (between the medial prefrontal cortex and the precuneus) (Jolles et al. 2013). However, the authors did not find these practice effects in a group of children (age 12, $n=$ 9), possibly due to sample size limitations resulting in a lack of power to find such effects. Nonetheless, the results of the aforementioned studies highlight the dynamic interaction between task-related brain regions and the precuneus, as well as the importance of connectivity between networks to support working memory task performance.

Table 2 Baseline to post-training changes in intra-network rsfc between the Cogmed and placebo groups

\begin{tabular}{|c|c|c|c|c|c|c|}
\hline $\begin{array}{l}\text { Intra-network resting state } \\
\text { functional connectivity }\end{array}$ & $\begin{array}{l}\text { Cogmed }(n=12) \\
\mathrm{M} \pm \mathrm{SD} \\
(z \text {-scores })\end{array}$ & $\begin{array}{l}\text { Placebo }(n=9) \\
\mathrm{M} \pm \mathrm{SD} \\
(z \text {-scores })\end{array}$ & Mean difference $^{\mathrm{a}}(95 \% \mathrm{CI})$ & Cohen's $d$ & $t$ & $p$ \\
\hline Precuneus network & $0.21 \pm 0.34$ & $0.03 \pm 0.47$ & $0.14(-0.22,0.51)$ & 0.44 & -0.82 & 0.42 \\
\hline Bilateral executive network & $0.10 \pm 0.48$ & $-0.03 \pm 0.24$ & $-0.001(-0.24,0.24)$ & 0.34 & 0.01 & 0.99 \\
\hline Dorsal attention network & $0.27 \pm 0.34$ & $-0.10 \pm 0.31$ & $0.27(0.02,0.52)$ & 1.14 & -2.23 & 0.04 \\
\hline Right central executive network & $0.04 \pm 0.40$ & $-0.16 \pm 0.23$ & $0.05(-0.19,0.29)$ & 0.61 & -0.44 & 0.66 \\
\hline Left central executive network & $0.12 \pm 0.31$ & $-0.07 \pm 0.37$ & $0.08(-0.15,0.30)$ & 0.56 & -0.72 & 0.48 \\
\hline Default mode network & $0.16 \pm 0.29$ & $-0.20 \pm 0.36$ & $0.25(-0.02,0.51)$ & 1.10 & -1.95 & 0.07 \\
\hline Salience network & $0.05 \pm 0.41$ & $-0.01 \pm 0.29$ & $0.02(-0.28,0.33)$ & 0.17 & -0.16 & 0.87 \\
\hline
\end{tabular}

${ }^{a}$ Mean differences were adjusted for baseline performance. The results did not survive multiple comparison correction. $z$-scores reflect the functional connectivity change within a network. Positive scores indicate higher functional connectivity at follow-up than at baseline, while negative scores indicate higher functional connectivity at baseline than at follow-up

$M$ mean, $S D$ standard deviation, $C I$ confidence interval 
Table 3 Baseline to post-training changes in inter-network rsfc between the Cogmed and placebo groups

\begin{tabular}{|c|c|c|c|c|c|c|}
\hline $\begin{array}{l}\text { Inter-network resting state } \\
\text { functional connectivity }\end{array}$ & $\begin{array}{l}\text { Cogmed }(n=12) \\
\mathrm{M} \pm \mathrm{SD} \\
(z \text {-scores })\end{array}$ & $\begin{array}{l}\text { Placebo }(n=9) \\
\mathrm{M} \pm \mathrm{SD} \\
(z \text {-scores })\end{array}$ & Mean difference $^{a}(95 \% \mathrm{CI})$ & Cohen's $d$ & $t$ & $p$ \\
\hline DMN-DAN & $-0.02 \pm 0.28$ & $-0.05 \pm 0.28$ & $-0.06(-0.27,0.17)$ & 0.11 & 0.52 & 0.61 \\
\hline DMN-LCEN & $0.09 \pm 0.24$ & $-0.05 \pm 0.15$ & $0.04(-0.09,0.17)$ & 0.70 & -0.66 & 0.52 \\
\hline DMN-RCEN & $-0.05 \pm 0.29$ & $-0.002 \pm 0.14$ & $-0.09(-0.27,0.09)$ & 0.21 & 1.06 & 0.30 \\
\hline DMN-SN & $0.01 \pm 0.26$ & $-0.02 \pm 0.36$ & $-0.05(-0.29,0.20)$ & 0.10 & 0.39 & 0.70 \\
\hline DMN-BEN & $-0.04 \pm 0.29$ & $-0.14 \pm 0.18$ & $0.004(-0.16,0.17)$ & 0.41 & -0.06 & 0.96 \\
\hline DMN-PN & $0.05 \pm 0.33$ & $-0.15 \pm 0.18$ & $0.16(-0.06 .0 .38)$ & 0.75 & -1.56 & 0.14 \\
\hline DAN-LCEN & $0.04 \pm 0.31$ & $-0.07 \pm 0.48$ & $-0.13(-0.37,0.11)$ & 0.27 & 1.15 & 0.27 \\
\hline DAN-RCEN & $-0.02 \pm 0.22$ & $-0.08 \pm 0.37$ & $-0.09(-0.28,0.09)$ & 0.20 & 1.05 & 0.31 \\
\hline DAN-SN & $0.01 \pm 0.29$ & $0.03 \pm 0.31$ & $-0.10(-0.33,0.12)$ & 0.07 & 0.97 & 0.35 \\
\hline DAN-BEN & $-0.02 \pm 0.19$ & $-0.15 \pm 0.20$ & $0.08(-0.08,0.25)$ & 0.67 & -1.06 & 0.30 \\
\hline DAN-PN & $-0.08 \pm 0.28$ & $0.02 \pm 0.18$ & $-0.12(-0.33,0.10)$ & 0.42 & 1.16 & 0.26 \\
\hline LCEN-RCEN & $-0.01 \pm 0.22$ & $-0.09 \pm 0.23$ & $-0.002(-0.18,0.17)$ & 0.36 & 0.03 & 0.98 \\
\hline LCEN-SN & $-0.10 \pm 0.22$ & $0.05 \pm 0.32$ & $-0.13(-0.35,0.08)$ & 0.55 & 1.32 & 0.20 \\
\hline LCEN-BEN & $-0.10 \pm 0.25$ & $-0.04 \pm 0.37$ & $-0.08(-0.32,0.15)$ & 0.19 & 0.75 & 0.47 \\
\hline LCEN-PN & $0.01 \pm 0.16$ & $-0.12 \pm 0.21$ & $0.06(-0.07,0.19)$ & 0.70 & -0.96 & 0.35 \\
\hline RCEN-SN & $-0.09 \pm 0.37$ & $0.01 \pm 0.43$ & $-0.20(-0.46,0.06)$ & 0.25 & 1.64 & 0.12 \\
\hline RCEN-BEN & $-0.04 \pm 0.20$ & $-0.03 \pm 0.32$ & $-0.09(-0.27,0.09)$ & 0.04 & 1.07 & 0.30 \\
\hline RCEN-PN & $-0.08 \pm 0.21$ & $0.04 \pm 0.21$ & $-0.11(-0.26,0.04)$ & 0.57 & 1.52 & 0.15 \\
\hline SN-BEN & $-0.08 \pm 0.21$ & $0.03 \pm 0.40$ & $-0.07(-0.23,0.09)$ & 0.34 & 0.93 & 0.36 \\
\hline SN-PN & $-0.03 \pm 0.29$ & $0.06 \pm 0.28$ & $-0.11(-0.35,0.14)$ & 0.32 & 0.89 & 0.39 \\
\hline BEN-PN & $-0.09 \pm 0.26$ & $-0.15 \pm 0.12$ & $0.04(-0.16,0.23)$ & 0.30 & -0.41 & 0.69 \\
\hline
\end{tabular}

${ }^{a}$ Mean differences were adjusted for baseline performance. $z$-scores refer to the functional connectivity change between two networks. Positive scores indicate higher functional connectivity at follow-up than at baseline, while negative scores indicate higher functional connectivity at baseline than at follow-up

$M$ mean, $S D$ standard deviation, $C I$ confidence interval, $P N$ precuneus network, $B E N$ bilateral executive network, $D A N$ dorsal attention network, $R C E N$ right central executive network, $L C E N$ left central executive network, $D M N$ default mode network, $S N$ salience network

The precuneus network is not commonly investigated in resting-state fMRI studies as it shows some overlap with the DMN. However, it has been suggested that the precuneus network may have a different role than the DMN, although the two networks converge with age, as shown in a crosslifespan dataset ( $N=126,7-85$ years old) (Yang et al. 2014). Therefore, it may be the case that the precuneus network is only distinguishable at a younger age, such as in extremely preterm children in our study. Another viewpoint is that the precuneus is not part of the DMN, as rsfc studies have found that the DMN terminates near the subparietal sulcus and exclusively involves the posterior cingulate cortex (Margulies et al. 2009; Buckner et al. 2008). Future studies in regard to the development of functional networks across age and specificity of the regions involved are needed.

In our study, although at a trend-level, greater improvements in the trained activities for the Cogmed group was associated with baseline to post-training rsfc changes within or between the following networks: (1) right central executive network, (2) right central executive and salience networks, and (3) left

Table 4 Baseline to post-training changes in working memory between the Cogmed and placebo groups

\begin{tabular}{llllll}
\hline & $\begin{array}{l}\text { Cogmed }(n=12) \\
\text { mean } \pm \text { SD }\end{array}$ & $\begin{array}{l}\text { Placebo }(n=9) \\
\text { mean } \pm \text { SD }\end{array}$ & $\begin{array}{l}\text { Mean difference } \\
\text { (95\% CI) }\end{array}$ & Cohen's $d$ \\
\hline Immediate memory composite score change $^{\mathrm{a}}$ & $2.41 \pm 1.73$ & $1.4 \pm 1.72$ & $-0.54(-2.06,0.98)$ & 0.59 & 0.52 \\
Working memory composite score change $^{\mathrm{a}}$ & $2.49 \pm 2.02$ & $1.1 \pm 2.35$ & $-0.41(-2.4,1.54)$ & 0.63 & 0.94 \\
Cogmed Improvement Index score & $19 \pm 7.03$ & - & & - \\
\hline
\end{tabular}

${ }^{a}$ Composite scores are $z$-scores; mean differences were adjusted for baseline performances

$S D$ standard deviation, $C I$ confidence interval 
Fig. 2 Scatter plots of the intranetwork rsfe changes in DAN and DMN. $r s f c$ resting state functional connectivity, $D A N$ dorsal attention network, $D M N$ default mode network. The middle line indicates the mean, with each line below and above indicating one standard deviation.
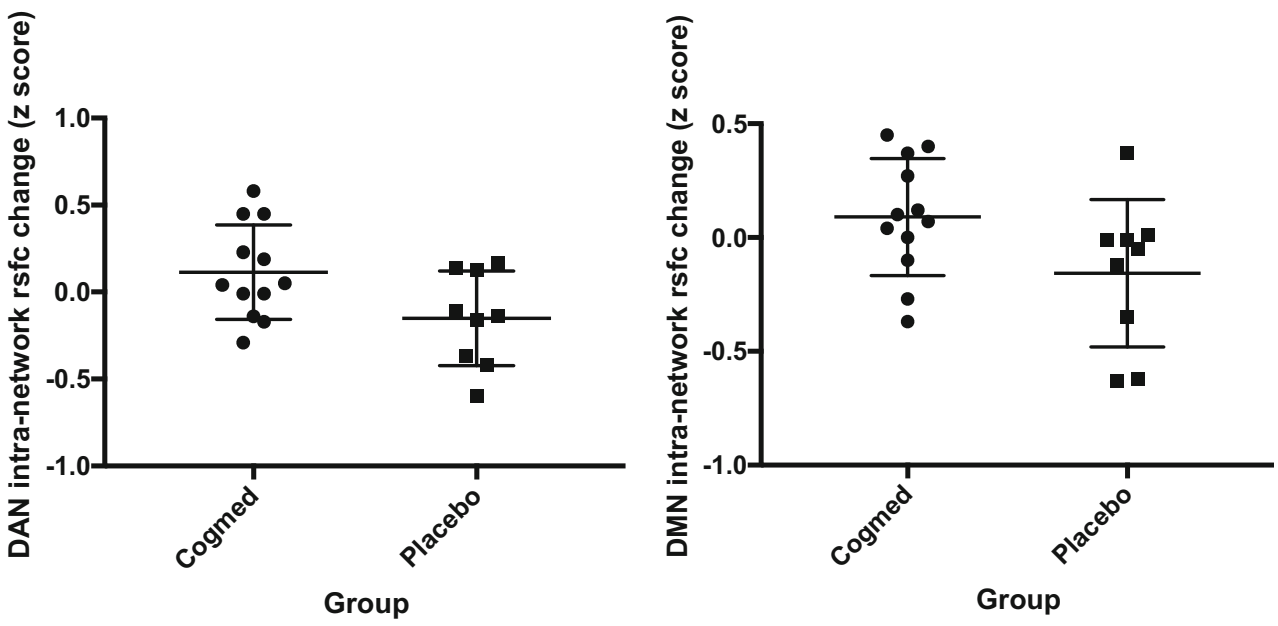

central executive and bilateral executive networks. The rsfc changes range from positive to negative values (i.e., a positive value means higher rsfc at follow-up than at baseline; a negative value means higher rsfc at baseline than at follow-up), which may reflect the shift in levels of cognitive effort in each individual. It is suggested that brain regions are initially more taxed when learning a new task, and with training, the task becomes less demanding and requires less activation (Buschkuehl et al. 2012). Therefore, the decrease of rsfe within the right central executive network along with Cogmed improvement possibly reflects reduced cognitive effort on working memory tasks post-training, as training may have increased children's proficiency at performing such tasks. This interpretation is similar to another study in very preterm children $(<$ 32 weeks' gestation), which observed decreased hemodynamic responses in frontoparietal regions during a visual working memory task and increased memory performance after memory strategy training and intensive working memory practice (Everts et al. 2015). Similarly, children with and without developmental dyscalculia showed improved performance and reduced recruitment of task-related brain regions during a spatial number representation task after mental number line training (Kucian et al. 2011). Decreased hemodynamic responses in task-related brain regions have also been commonly reported in working memory training studies in adolescents and adults (Buschkuehl et al. 2012). Taken together with our findings, it seems that increased efficiency of working memory processes occurs after training, resulting in decreased recruitment of taskrelated brain regions (frontal and parietal regions).

Similar to our study, a randomized controlled trial assessed the effects of Cogmed compared with a placebo program on functional connectivity, but in typically developing children and using magnetoencephalography rather than fMRI. The study found training-induced rsfc increases between
Fig. 3 Association between training-induced change in working memory composite score and precuneus network intra-network rsfc change, in the Cogmed and placebo groups. $P N$ precuneus network, $r s f c$ resting state functional connectivity.

\section{Working memory composite score change and PN intra-network rsfc change}

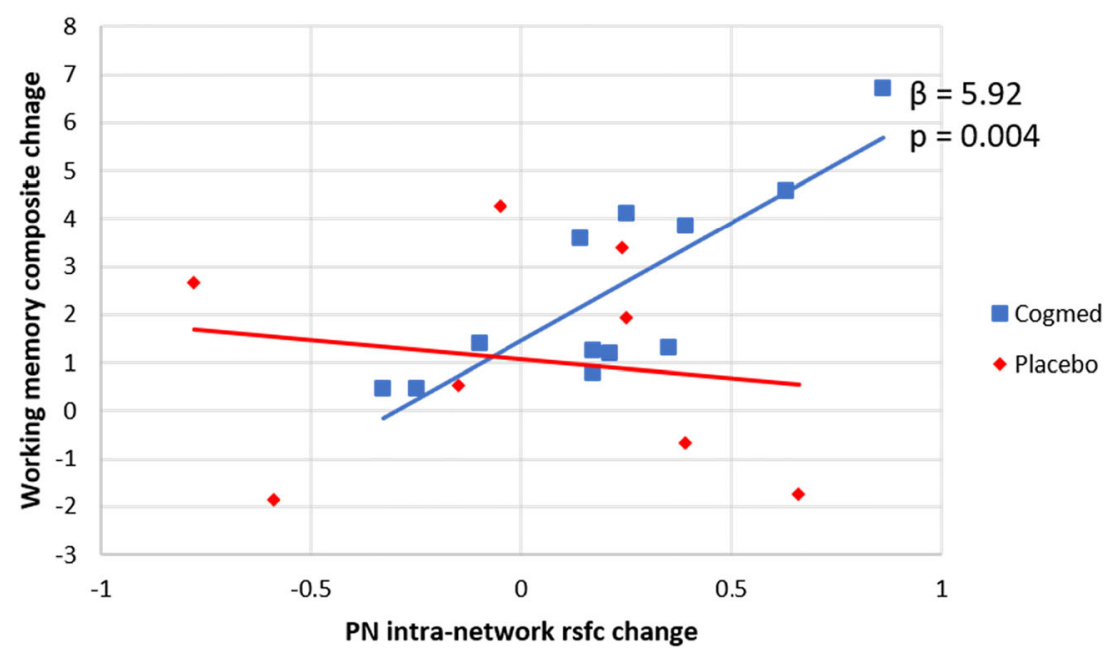



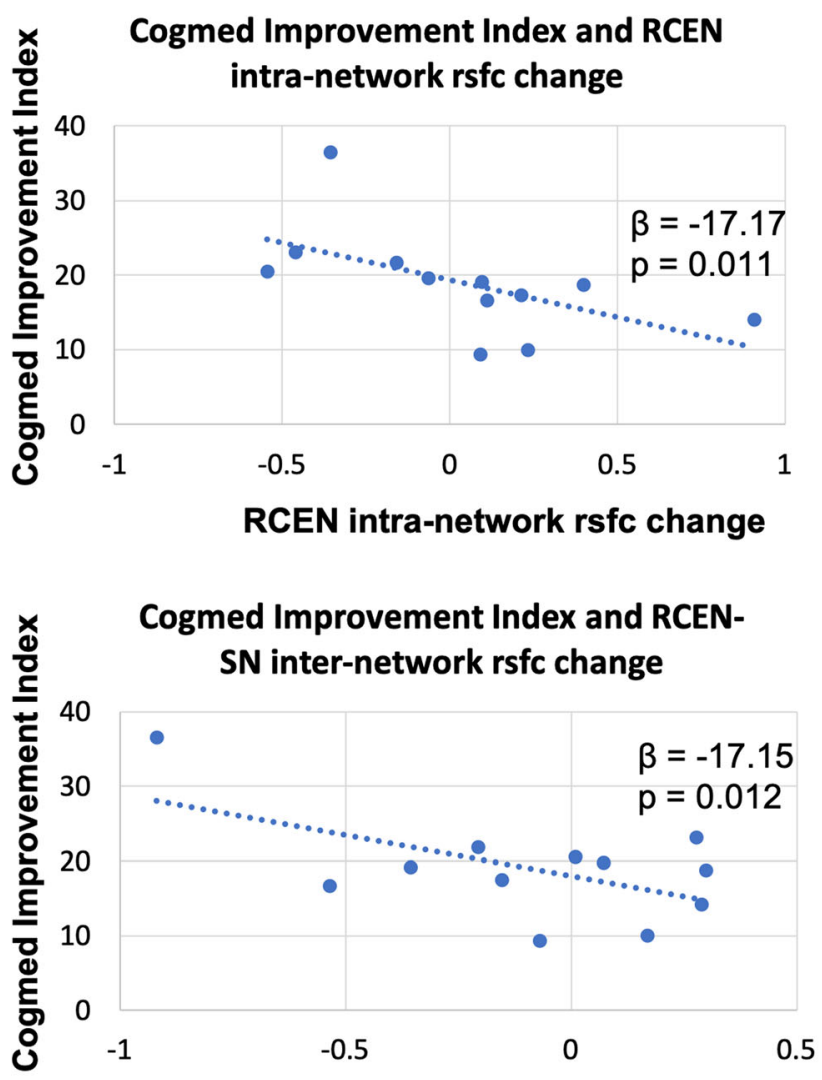

RCEN-SN inter-network rsfc change

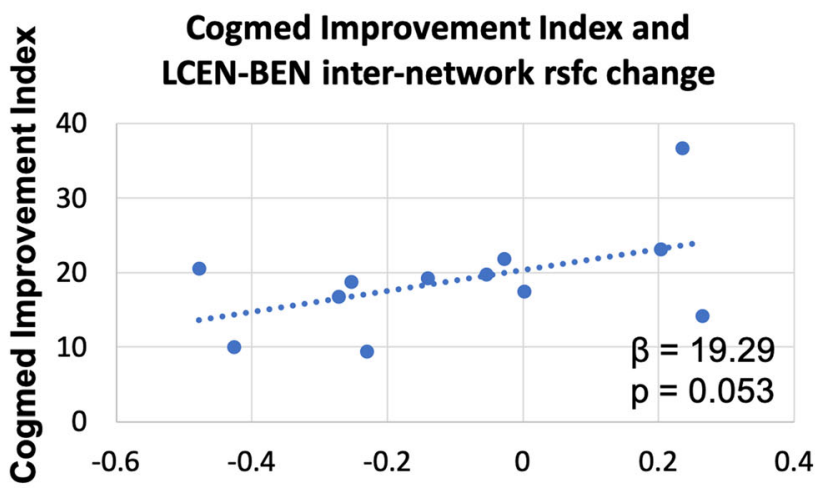

LCEN-BEN inter-network rsfc change

Fig. 4 Cogmed Improvement Index and resting state functional connectivity (rsfc) changes. RCEN right central executive network, $S N$ salience network, $L C E N$ left central executive network, $B E N$ bilateral central executive network

frontoparietal networks and other cortical areas, with working memory gains mirroring increases in rsfc (Astle et al. 2015). Our findings showed both increased and decreased internetwork connectivity in relation to improvement in trained activities, possibly highlighting the dynamic interaction between networks (although not surviving multiple comparison correction). These Cogmed-specific findings could be attributed to the adaptive nature of the Cogmed working memory training program. It is possible that adaptive training is more likely to induce brain-behavior changes compared with nonadaptive training. However, the causal relationship between adaptive training, working memory, and rsfe cannot be inferred from this study and warrants future research.

Greater intra-network rsfc changes in the DAN and DMN were found, at a trend-level, in the Cogmed group compared to the placebo group. However, rsfc changes in neither of these two networks were associated with working memory changes in this study, demonstrating that differences in rsfc changes between training groups can occur without associated changes in behavioral manifestation (Pascual-Leone et al. 2005). It is also possible that the behavioral changes occurred in other untested domains.

Ours is the first study to investigate the effects of Cogmed on brain function in EP/ELBW children. Using a functional network perspective, we were able to evaluate changes in network rsfc strength and interactions, rather than limiting to regional rsfc changes. Associations between training-induced rsfe change and changes in trained activities for only the Cogmed group were found, despite little evidence of group differences between the Cogmed and placebo groups in rsfc and working memory change. This is contrary to our hypothesis that associations between training-induced rsfc change and improvements in trained activities would be similar regardless of training group and suggests the importance of exploring within group neural-cognitive associations.

The current study was limited by sample size, and therefore, findings need to be interpreted with caution and reevaluated with a larger sample. It is important to note that there was little evidence for differences in working memory between the Cogmed and placebo groups at 24 months posttraining in the main trial which may also have limited our power (Anderson et al. 2018). It remains to be ascertained whether EP/ELBW children require a longer training period or more training sessions to result in greater behavioral effects (baseline to post-training working memory gains), due to reduced neuroplasticity (Pitcher et al. 2012). It should be noted that the Cogmed Improvement Index score, which we used to assess improvement in trained activities, does not necessarily reflect the final effect of training as the Max index is not necessarily from the last trial. This score may better represent the capacity of improvement across training trials. Additionally, especially in children, subject motion is an issue that may affect functional connectivity measures. In our analyses we used well-established ICA-based denoising methods that have been shown to be effective in reducing spatially specific motion artifacts and also some global artifacts, the two main sources of motion artifacts in resting-state data (Burgess et al. 2016).

The current study demonstrates that working memory performance following Cogmed was associated with selective functional connectivity changes in EP/ELBW children. Further studies are needed to better understand whether more 
extensive training, possibly eliciting greater neuroanatomical changes, can lead to behavioral improvements and support cognitive growth in vulnerable groups.

Acknowledgments This research was conducted within the Developmental Imaging research group, Murdoch Children's Research Institute and the Children's MRI Centre, Royal Children's Hospital, Melbourne, Victoria. We thank all participating children and families who made this research possible. We thank the Royal Children's Hospital Medical Imaging staff for their assistance and expertise in the collection of the MRI data included in this study.

Funding Information This study was supported by the Murdoch Children's Research Institute, the Royal Children's Hospital, The Royal Children's Hospital Foundation, the Department of Paediatrics at The University of Melbourne, Monash University, and the Victorian Government's Operational Infrastructure Support Program. This study was also supported by the Australian National Health and Medical Research Council (NHMRC: Project Grant 1028422, Centre of Research Excellence in Newborn Medicine (1060733), Program Grant 606789, Senior Research Fellowship 1081288, Career Development Fellowship 1085754). This article was made open access with the financial support of King's College London.

\section{Compliance with Ethical Standards}

All procedures performed in studies involving human participants were in accordance with the ethical standards of the institutional and/or national research committee and with the 1964 Helsinki declaration and its later amendments or comparable ethical standards. Informed consent was obtained from all individual participants included in the study.

Conflict of Interest The authors declare that they have no conflict of interest.

Open Access This article is distributed under the terms of the Creative Commons Attribution 4.0 International License (http:// creativecommons.org/licenses/by/4.0/), which permits unrestricted use, distribution, and reproduction in any medium, provided you give appropriate credit to the original author(s) and the source, provide a link to the Creative Commons license, and indicate if changes were made.

\section{References}

Alloway, T. P. (2007). Automated working memory assessment. London: Pearson Assessment.

Anderson, P. J. (2014). Neuropsychological outcomes of children born very preterm. Seminars in Fetal \& Neonatal Medicine, 19, 90-96.

Anderson, P. J., \& Doyle, L. W. (2008). Cognitive and educational deficits in children born extremely preterm. Seminars in Perinatology, $32,51-58$.

Anderson, P. J., Lee, K. J., Roberts, G., Spencer-Smith, M. M., Thompson, D. K., Seal, M. L., Nosarti, C., Grehan, A., Josev, E. K., Gathercole, S., Doyle, L. W., \& Pascoe, L. (2018). Long-term academic functioning following Cogmed working memory training for children born extremely preterm: A randomized controlled trial. The Journal of Pediatrics, 202, 92-97 e94.
Andersson, J.L.R., Jenkinson, M., Smith, S. (2010). Non-linear registration, aka spatial normalisation. FMRIB technical report TR07JA2.

Astle, D. E., Barnes, J. J., Baker, K., Colclough, G. L., \& Woolrich, M. W. (2015). Cognitive training enhances intrinsic brain connectivity in childhood. The Journal of Neuroscience : the Official Journal of the Society for Neuroscience, 35, 6277-6283.

Bell, A. J., \& Sejnowski, T. J. (1995). An information maximization approach to blind separation and blind deconvolution. Neural Computation, 7, 1129-1159.

Benjamini, Y., \& Hochberg, Y. (1995). Controlling the false discovery ratea practical and powerful approach to multiple testing. Journal of the Royal Statistical Society. Series B, Methodological, 57, 289-300.

Buckner, R. L., Andrews-Hanna, J. R., \& Schacter, D. L. (2008). The brain's default network: Anatomy, function, and relevance to disease. Annals of the New York Academy of Sciences, 1124, 1-38.

Burgess, G. C., Kandala, S., Nolan, D., Laumann, T. O., Power, J. D., Adeyemo, B., Harms, M. P., Petersen, S. E., \& Barch, D. M. (2016). Evaluation of denoising strategies to address motioncorrelated artifacts in resting-state functional magnetic resonance imaging data from the human connectome project. Brain Connectivity, 6 , $669-680$

Buschkuehl, M., Jaeggi, S. M., \& Jonides, J. (2012). Neuronal effects following working memory training. Developmental Cognitive Neuroscience, 2, S167-S179.

Calhoun, V. D., Kiehl, K. A., \& Pearlson, G. D. (2008). Modulation of temporally coherent brain networks estimated using ICA at rest and during cognitive tasks. Human Brain Mapping, 29, 828-838.

Cavanna, A. E., \& Trimble, M. R. (2006). The precuneus: A review of its functional anatomy and behavioural correlates. Brain., 129, 564-583.

Charroud, C., Le Bars, E., Deverdun, J., Steffener, J., Molino, F., Abdennour, M., Portet, F., Bonafe, A., Stern, Y., Ritchie, K., Akbaraly, T. N., \& de Champfleur, N. M. (2016). Working memory performance is related to intrinsic resting state functional connectivity changes in community-dwelling elderly cohort. Neurobiology of Learning and Memory, 132, 57-66.

Doyle, L. W., Roberts, G., Anderson, P. J., \& Victorian Infant Collaborative Study G. (2010). Outcomes at age 2 years of infants $<28$ weeks' gestational age born in Victoria in 2005. The Journal of Pediatrics., 156, 49-53 e41.

Doyle, L. W., Roberts, G., Anderson, P. J., \& Victorian Infant Collaborative Study G. (2011). Changing long-term outcomes for infants 500-999 g birth weight in Victoria, 1979-2005. Archives of Disease in Childhood. Fetal and Neonatal Edition, 96, F443-F447.

Engstrom, M., Landtblom, A. M., \& Karlsson, T. (2013). Brain and effort: Brain activation and effort-related working memory in healthy participants and patients with working memory deficits. Frontiers in Human Neuroscience, 7, 140.

Erk, S., Spottke, A., Meisen, A., Wagner, M., Walter, H., \& Jessen, F. (2011). Evidence of neuronal compensation during episodic memory in subjective memory impairment. Archives of General Psychiatry, 68, 845-852.

Everts, R., Murner-Lavanchy, I., Schroth, G., \& Steinlin, M. (2015). Neural change following different memory training approaches in very preterm born children-a pilot study. Developmental Neurorehabilitation, 20, 14-24.

Fang, X. J., Zhang, Y. C., Zhou, Y., Cheng, L. Q., Li, J., Wang, Y. L., Friston, K. J., \& Jiang, T. Z. (2016). Resting-state coupling between core regions within the central-executive and salience networks contributes to working memory performance. Frontiers in Behavioral Neuroscience, 10:27, 1-11.

Froudist-Walsh, S., Karolis, V., Caldinelli, C., Brittain, P. J., Kroll, J., Rodriguez-Toscano, E., Tesse, M., Colquhoun, M., Howes, O., 
Dell'Acqua, F., de Schotten, M. T., Murray, R. M., Williams, S. C. R., \& Nosarti, C. (2015). Very early brain damage leads to remodeling of the working memory system in adulthood: A combined fMRI/tractography study. The Journal of Neuroscience, 35, 15787-15799.

Gathercole, S. E. (1999). Cognitive approaches to the development of short-term memory. Trends in Cognitive Sciences, 3, 410-419.

Griffanti, L., Salimi-Khorshidi, G., Beckmann, C. F., Auerbach, E. J., Douaud, G., Sexton, C. E., Zsoldos, E., Ebmeier, K. P., Filippini, N., Mackay, C. E., Moeller, S., Xu, J., Yacoub, E., Baselli, G., Ugurbil, K., Miller, K. L., \& Smith, S. M. (2014). ICA-based artefact removal and accelerated fMRI acquisition for improved resting state network imaging. Neuroimage., 95, 232-247.

Grunewaldt, K. H., Lohaugen, G. C., Austeng, D., Brubakk, A. M., \& Skranes, J. (2013). Working memory training improves cognitive function in VLBW preschoolers. Pediatrics., 131, e747-e754.

Hampson, M., Driesen, N. R., Skudlarski, P., Gore, J. C., \& Constable, R. T. (2006). Brain connectivity related to working memory performance. The Journal of Neuroscience: the Official Journal of the Society for Neuroscience, 26, 13338-13343.

Heath, N. L., \& Ross, S. (2000). Prevalence and expression of depressive symptomatology in students with and without learning disabilities. Learning Disability Quarterly, 23, 24-36.

Himberg, J., Hyvarinen, A., \& Esposito, F. (2004). Validating the independent components of neuroimaging time series via clustering and visualization. NeuroImage., 22, 1214-1222.

Holmes, J., Gathercole, S. E., \& Dunning, D. L. (2009). Adaptive training leads to sustained enhancement of poor working memory in children. Developmental Science, 12, F9-F15.

Jenkinson, M., \& Smith, S. (2001). A global optimisation method for robust affine registration of brain images. Medical Image Analysis, $5,143-156$

Jenkinson, M., Bannister, P., Brady, M., \& Smith, S. (2002). Improved optimization for the robust and accurate linear registration and motion correction of brain images. Neuroimage., 17, 825-841.

Johnson, M. H. (2011). Interactive specialization: A domain-general framework for human functional brain development? Developmental Cognitive Neuroscience, 1, 7-21.

Jolles, D. D., \& Crone, E. A. (2012). Training the developing brain: A neurocognitive perspective. Frontiers in Human Neuroscience, 6, 76.

Jolles, D. D., van Buchem, M. A., Crone, E. A., \& Rombouts, S. A. R. B. (2013). Functional brain connectivity at rest changes after working memory training. Human Brain Mapping, 34, 396-406.

Kerns, K. A., Macoun, S., MacSween, J., Pei, J., \& Hutchison, M. (2017). Attention and working memory training: A feasibility study in children with neurodevelopmental disorders. Applied Neuropsychology: Child, 6, 120-137.

Klingberg, T., Forssberg, H., \& Westerberg, H. (2002). Training of working memory in children with ADHD. Journal of Clinical and Experimental Neuropsychology, 24, 781-791.

Klingberg, T., Fernell, E., Olesen, P. J., Johnson, M., Gustafsson, P., Dahlstrom, K., Gillberg, C. G., Forssberg, H., \& Westerberg, H, (2005). Computerized training of working memory in children with ADHD-a randomized, controlled trial. Journal of the American Academy of Child and Adolescent Psychiatry, 44, 177-186.

Kucian, K., Grond, U., Rotzer, S., Henzi, B., Schonmann, C., Plangger, F., Galli, M., Martin, E., \& von Aster, M. (2011). Mental number line training in children with developmental dyscalculia. NeuroImage., 57, 782-795.

Levine, P., \& Nourse, S. W. (1998). What follow-up studies say about postschool life for young men and women with learning disabilities:
A critical look at the literature. Journal of Learning Disabilities, 31, 212-233.

Lewis, C. M., Baldassarre, A., Committeri, G., Romani, G. L., \& Corbetta, M. (2009). Learning sculpts the spontaneous activity of the resting human brain. Proceedings of the National Academy of Sciences of the United States of America, 106, 17558-17563.

Lohaugen, G. C., Antonsen, I., Haberg, A., Gramstad, A., Vik, T., Brubakk, A. M., \& Skranes, J. (2011). Computerized working memory training improves function in adolescents born at extremely low birth weight. The Journal of Pediatrics, 158, 555-561 e554.

Margulies, D. S., Vincent, J. L., Kelly, C., Lohmann, G., Uddin, L. Q., Biswal, B. B., Villringer, A., Castellanos, F. X., Milham, M. P., \& Petrides, M. (2009). Precuneus shares intrinsic functional architecture in humans and monkeys. Proceedings of the National Academy of Sciences of the United States of America, 106, 20069-20074.

Marlow, N. (2004). Neurocognitive outcome after very preterm birth. Archives of Disease in Childhood. Fetal and Neonatal Edition, 89, F224-F228.

Mennes, M., Kelly, C., Zuo, X. N., Di Martino, A., Biswal, B. B., Castellanos, F. X., \& Milham, M. P. (2010). Inter-individual differences in resting-state functional connectivity predict task-induced BOLD activity. NeuroImage., 50, 1690-1701.

Nikolaidis, A., Voss, M. W., Lee, H., Vo, L. T. K., \& Kramer, A. F. (2014). Parietal plasticity after training with a complex video game is associated with individual differences in improvements in an untrained working memory task. Frontiers in Human Neuroscience, 8:169, 111.

Pascual-Leone, A., Amedi, A., Fregni, F., \& Merabet, L. B. (2005). The plastic human brain cortex. Annual Review of Neuroscience, 28, 377-401.

Pickering, S., \& Gathercole, S. (2001). Working memory test battery for children-manual. London: The Psychological Corporation.

Pitcher, J. B., Riley, A. M., Doeltgen, S. H., Kurylowicz, L., Rothwell, J. C., McAllister, S. M., Smith, A. E., Clow, A., Kennaway, D. J., \& Ridding, M. C. (2012). Physiological evidence consistent with reduced neuroplasticity in human adolescents born preterm. The Journal of Neuroscience : the Official Journal of the Society for Neuroscience., 32, 16410-16416.

Robinson, K. E., Kaizar, E., Catroppa, C., Godfrey, C., \& Yeates, K. O. (2014). Systematic review and meta-analysis of cognitive interventions for children with central nervous system disorders and neurodevelopmental disorders. Journal of Pediatric Psychology, 39, 846-865.

Sala-Llonch, R., Pena-Gomez, C., Arenaza-Urquijo, E. M., Vidal-Pineiro, D., Bargallo, N., Junque, C., \& Bartres-Faz, D. (2012). Brain connectivity during resting state and subsequent working memory task predicts behavioural performance. Cortex: a Journal Devoted to the Study of the Nervous System and Behavior, 48, 1187-1196.

Salimi-Khorshidi, G., Douaud, G., Beckmann, C. F., Glasser, M. F., Griffanti, L., \& Smith, S. M. (2014). Automatic denoising of functional MRI data: Combining independent component analysis and hierarchical fusion of classifiers. Neuroimage., 90, 449-468.

Shirer, W. R., Ryali, S., Rykhlevskaia, E., Menon, V., \& Greicius, M. D. (2012). Decoding subject-driven cognitive states with whole-brain connectivity patterns. Cerebral Cortex, 22, 158-165.

Simone, A. N., Marks, D. J., Bédard, A.-C., \& Halperin, J. M. (2017). Low working memory rather than ADHD symptoms predicts poor academic achievement in school-aged children. Journal of Abnormal Child Psychology, 46(2), 277-290.

Smith, S. M., Fox, P. T., Miller, K. L., Glahn, D. C., Fox, P. M., Mackay, C. E., Filippini, N., Watkins, K. E., Toro, R., Laird, A. R., \& Beckmann, C. F. (2009). Correspondence of the brain's functional 
architecture during activation and rest. Proceedings of the National Academy of Sciences of the United States of America, 106, 1304013045.

Utevsky, A. V., Smith, D. V., \& Huettel, S. A. (2014). Precuneus is a functional core of the default-mode network. The Journal of Neuroscience, 34, 932-940.

Yang, Z., Chang, C., Xu, T., Jiang, L., Handwerker, D. A., Castellanos, F. X., Milham, M. P., Bandettini, P. A., \& Zuo, X. N. (2014). Connectivity trajectory across lifespan differentiates the precuneus from the default network. Neuroimage., 89, 45-56.
Zou, Q., Ross, T. J., Gu, H., Geng, X., Zuo, X. N., Hong, L. E., Gao, J. H., Stein, E. A., Zang, Y. F., \& Yang, Y. (2013). Intrinsic resting-state activity predicts working memory brain activation and behavioral performance. Human Brain Mapping, 34, 3204-3215.

Publisher's Note Springer Nature remains neutral with regard to jurisdictional claims in published maps and institutional affiliations. 